\title{
ICNOFÁBRICA DE Ophiomorpha/Conichnus EN EL MIOCENO INFERIOR DE CRISTO REI (ALMADA, PORTUGAL). IMPLICACIONES PALEOAMBIENTALES
}

\author{
Jordi Maria de GIBERT', Carlos Marques da SILVA² y \\ Mario $C A C H A O^{2}$
} ' Department of Geology and Geophysics. University of Utah. 135 South 1460 East.
Room 719. Salt Lake City, UT 84112-1183, U.S.A.
2 Dpto. de Geologia, Universidade de Lisboa. Rua da Escola Politécnica 58. P-1294
Lisboa Codex, Portugal.

Gibert, J.M. de, Silva, C.M. da y Cachao, M. 1998. Icnofábrica de OphiomorphalConichnus en el Mioceno Inferior de Cristo Rei (Almada, Portugal). Implicaciones paleoambientales. [Ophiomorpha/Conichnus ichnofabric in the Lower Miocene of Cristo Rei (Almada, Portugal). Palaeoenvironmental implications]. Revista Española de Paleontología, 13 (2), 251-259. ISSN 0213-6937.

\begin{abstract}
Ophiomorpha nodosa and Conichnus conicus are described from the Burdigalian (Lower Miocene) of the CristoRei outcrop in Almada, near Lisbon (Portugal). These bioturbation structures form an ichnofabric with a low to moderate bioturbation index. Sedimentary structures, mostly horizontal and cross lamination, are dominant over the biogenic structures. The trace fossil assemblage, together with the characteristics of the ichnofabric, allow us to suggest that the depositional setting for the sandstones was a shallow marine environment with high sedimentation rate and high bottom energy, probably a sand shoal in an estuarine setting. The same ichnofabric has been described from similar deposits of different ages and places, and so it has an important value as a palaeoenvironmental indicator.
\end{abstract}

Keywords: Trace fossils, Conichnus, Ophiomorpha, Miocene, shallow marine, Portugal.

\section{RESUMEN}

Se describen Ophiomorpha nodosa y Conichnus conicus de las areniscas del Burdigaliense (Mioceno Inferior) del afloramiento de Cristo-Rei en Almada (Portugal). La icnofábrica a que dan lugar presenta un índice de bioturbación bajo a moderado. Las estructuras sedimentarias (laminaciones paralelas y cruzadas) son dominántes sobre las biogénicas. La asociación de trazas fósiles junto con las características de la icnofábrica sugieren un medio de depósito marino somero, con elevada tasa de sedimentación y alta energía en el fondo, probablemente en una zona de bancos arenosos en un contexto estuarino. La misma icnofábrica es conocida en depósitos similares de otras edades y áreas geográficas, lo que le confiere un importante valor como indicador paleoambiental.

Palabras clave: Icnofósiles, Conichnus, Ophiomorpha, Mioceno, marino somero, Portugal.

\section{INTRODUCCIÓN}

Las publicaciones sobre estudios icnológicos realizados en Portugal son escasas. Únicamente la icnología de vertebrados, en particular la de dinosaurios, ha merecido una especial atención (e.g. Santos et al., 1994; Lockley et al., 1994; Dantas et al., 1994; Meyer et al., 1994). Por lo que respecta a los trabajos relativos a icnología de invertebrados, cabe destacar los de Cooper y Romano (1982) en el Ordovícico, Fürsich (1981) en el Jurásico, o más recientemente, Silva et al. (1995) en bioerosión del Neógeno.
En el presente trabajo se aborda el estudio de la bioturbación en el Burdigaliense (Mioceno Inferior) del afloramiento de Cristo Rei (Almada), en el margen izquierdo del río Tajo, frente a la ciudad de Lisboa. Esta contribución constituye el primer trabajo sobre bioturbación del Neógeno port ugués y con ella se pretende poner de manifiesto la utilidad del análisis de icnofábricas para la reconstrucción de paleoambientes.

El uso de icnofósiles en reconstrucciones paleoambientales no debe fundamentarse únicamente en la presencia de determinados icnotaxones, sino que se debe basar en el estudio de asociaciones de icnofósiles 


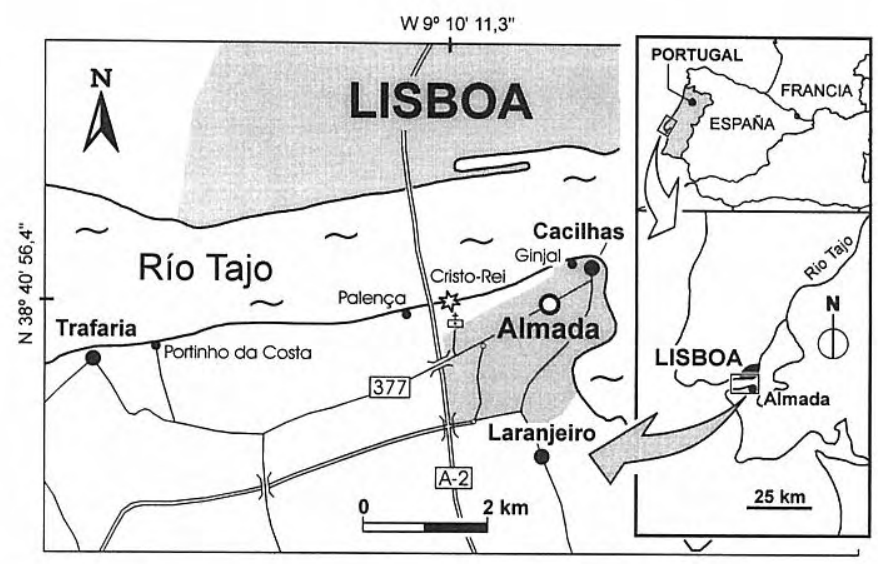

Figura 1. Localización geográfica del perfil de Cristo-Rei.

(icnoasociaciones). Las conocidas icnofacies de Seilacher (1964, 1967; Ekdale et al., 1984) -icnoasociaciones recurrentes en el tiempo y el espacio características de determinadas condiciones batimétricas- fueron una primera e importantísima aportación que ha sido de gran utilidad por su pretendida validez universal. Sin embargo, en los últimos años, los progresos en el campo de la sedimentología y en la propia icnología han evidenciado el carácter excesivamente generalista de las icnofacies y la necesidad de un grado de resolución mayor (Ekdale, 1988; Bromley y Asgaard, 1991; Goldring, 1993). El estudio y caracterización de icnoasociaciones locales permite una mayor concreción en la caracterización de ambientes y subambientes, así como la comparación entre localidades de diferente edad y localización geográfica. Recientemente, el nuevo concepto de icnofábrica (definido por Ekdale y Bromley, 1983) ha supuesto un paso más en el uso de las pistas fósiles en sedimentología y reconstrucciones paleoambientales. El concepto de icnofábrica es más complejo que el de icnoasociación puesto que incluye no sólo el conjunto de icnofósiles presente en un depósito sino también la relación entre ellos y con las estructuras sedimentarias, su modo de conservación y la intensidad de bioturbación (Taylor y Goldring, 1993).

\section{MARCO GEOGRÁFICO Y GEOLÓGICO}

El corte de Cristo-Rei se sitúa en el margen izquierdo del río Tajo, al sur de Lisboa, en Pragal (Almada), inmediatamente bajo el monumento del mismo nombre, en un lugar conocido antiguamente como "Forno do Tijolo". En este sector, el estuario del Tajo se estrecha originando el denominado "Gargalo do Tejo", que se encuentra bordeado al sur por un extenso acantilado, con una altura máxima de 125 metros, constituído por rocas fosilíferas de edad miocena (Fig. 1).

Desde el punto de vista geológico, la secuencia miocena de Cristo-Rei forma parte de la unidad morfoestructural meso-cenozoica denominada Cuenca del Bajo Tajo y Sado (Ribeiro et al., 1979). En la región de Almada-Trafaria, la secuencia es monoclinal con un ligero buzamiento hacia el SSE, constituyendo el flanco norte del sinclinal de Albufeira, que limita al norte con una estructura anticlinal alpina que constituye la Serra de Arrábida, localizada a unos $30 \mathrm{Km}$ al sur de Lisboa.

\section{ESTRATIGRAFÍA}

\section{ANTECEDENTES}

El estudio de los afloramientos cenozoicos del margen izquierdo del Tajo al sur de Lisboa (Península de Setúbal) se remonta, por lo menos, a la primera mitad del siglo pasado, cuando von Eschwege (1831) los incluyó en la denominada "Formación Terciaria" que englobaba afloramientos de Lisboa, Almada y la Serra de Arrábida, más al sur. Poco después, Sharpe (1834), en su estudio de la "gran cuenca terciaria" de los alrededores de Lisboa, incluye los terrenos de Almada (donde se encuentra el corte de Cristo-Rei) en la unidad designada por él como las "capas de Almada" y les atribuye una edad terciaria indiferenciada. Es Smith (1847) quien, basándose en el estudio de la malacofauna, atribuye por primera vez una edad miocena a estos afloramientos. Cotter (1956), a finales del siglo pasado y principios de éste, define y describe un conjunto de unidades litoestratigráficas (unidades I a VII de Cotter), inicialmente con significado cronoestratigráfico. El corte estudiado aquí corresponde a lo que, generalmente, es considerado la transición entre las unidades II y III de Cotter, atribuídas por él al Burdigaliense medio (Dollfus et al., 1903-1904).

\section{EL CORTE DE CRISTO-REI}

El corte de Cristo-Rei constituye una secuencia sedimentaria terrígena de edad miocena, con alrededor de 100 metros de potencia, formada esencialmente por limolitas, areniscas finas a gruesas y, en menor grado, arcillas. En los primeros dos o tres metros basales de la secuencia, junto al río, se reconoce una superficie irregular con un truncamiento erosivo, que separa la capa bioturbada estudiada (Fig. 2, capa 1) de la capa fosilífera suprayacente (capa 2) (Lám. I, fig. 1). Este contacto erosivo es observable, por lo menos, desde el Ginjal (Almada) hasta el Portinho da Costa (Trafaria) (Fig. 1). Según Antunes (in Pais, 1981, p. 41) y Antunes et al. (1992), este truncamiento define el límite entre dos de las unidades clásicas de Cotter (1956): la unidad II ("Areolas de Pecten pseudo-pandorae da Avenida Estefània") y la unidad III ("Banco Real").

En Cristo-Rei, el techo de la unidad II (Fig. 2, capa 1) está constituído por areniscas de grano medio a grueso, de color marrón claro, con cemento carbonatado, incluyendo niveles de laminación paralela y cruzada, bioturbadas (Ophiomorpha nodosa y Conichnus conicus) y con algunos restos de macroinvertebrados, principalmente pinzas de cangrejos, fragmentos de equinoideos, placas marginales de asteroideos y bivalvos (ostréidos). La base de la Unidad III (Fig. 2, capa 2) está constituída por areniscas de grano fino, color gris claro a marrón rojizo (por alteración), con cemento carbonatado y abundantes fósiles de macroinvertebrados, incluyendo gasterópodos (principalmente turritélidos), bivalvos (principalmente pectínidos y 
ostréidos), fragmentos de equinoideos (clypeasteroideos) y briozoos.

\section{BIOESTRATIGRAFÍA}

\section{INTRODUCCIÓN}

Según Antunes et al. (1992), los niveles superiores de la Unidad II de Cotter, en la región de Lisboa, suministran una asociación de foraminíferos planctónicos (Globigerinoides primordius Blow y Banner y $G$. subquadratus Brönnimann; cf. Antunes y Mein, 1986) característica de la biozona N5 de Blow (1969), que corresponde aproximadamente al Burdigaliense inferior. El estudio de Fonseca (1977) de las asociaciones de nanofósiles calcáreos del corte de Palença, próximo a Cristo-Rei (Fig. 1), abarcando las Unidades II a Vb de Cotter, no es conclusivo respecto la posición de las capas de la Unidad II. Sólo se da con fuertes reservas una atribución a la biozona NN4 de Martini (1971) (Burdigaliense superior-Langhiense) para los niveles superiores del corte de Palença (Unidad IV).

\section{EL CORTE DE CRISTO-REI}

El estudio de las muestras recogidas en las capas 1 (techo de la Unidad II de Cotter) y 2 (base de la unidad III) del Corte de Cristo-Rei revela las siguientes asociaciones de nanofósiles calcáreos:

Capa 1: Coccolithus pelagicus (Wallich) Schiller, Cyclicargolithus floridanus (Roth y Hay) Bukry, Dictyococcites antarticus Haq, D. productus (Kamptner) Backman, Geminilithella jaffari (Müller) Backman, Helicosphaera carteri (Wallich) Kamptner, Reticulofenestra minuta Roth, R. gr. haqii Backman - minutula Haq y Berggren, Sphenolithus sp.

Capa 2: Calcidiscus leptoporus (Murray y Blackman) Loeblich y Tappan, Coccolithus pelagicus, Coronocyclus nitescens (Kamptner) Bramlette y Wilcoxon, Cyclicargolithus floridanus (Roth y Hay) Bukry, Dictyococcites antarticus Haq, D. productus (Kamptner) Backman, Geminilithella jaffari (Müller) Backman, G. rotula (Kamptner) Backman, Helicosphaera ampliaperta Bramlette y Wilcoxon, $H$. carteri, $H$. mediterranea Müller, $H$. vedderi Bukry, Reticulofenestra minuta Roth, R. gr. haqii Backman minutula Haq y Berggren, Sphenolithus heteromorphus Deflandre, Syracosphaera sp.

La poca abundancia y diversidad de los nanolitos de la capa 1 condujo al muestreo de un corte adicional en el Portinho da Costa (al oeste de Cristo-Rei, Fig. 1), donde se reconoce la misma superficie de discontinuidad y la Unidad II está mejor representada, ya que afloran 13 o 14 metros, permitiendo estudiar niveles estratigráficamente más bajos que en Cristo-Rei. De manera similar a Cristo-Rei, en Portinho da Costa el techo de la unidad está constituído por areniscas de grano medio a grueso, con alternancia de laminación paralela y estratificación cruzada, bioturbadas (con estructuras biogénicas peor conservadas que en CristoRei: ?Ophiomorpha isp.) y con raros restos de invertebrados. Así, en Portinho da Costa la asociación encontrada en los niveles más bajos de la Unidad II de Cotter incluye:

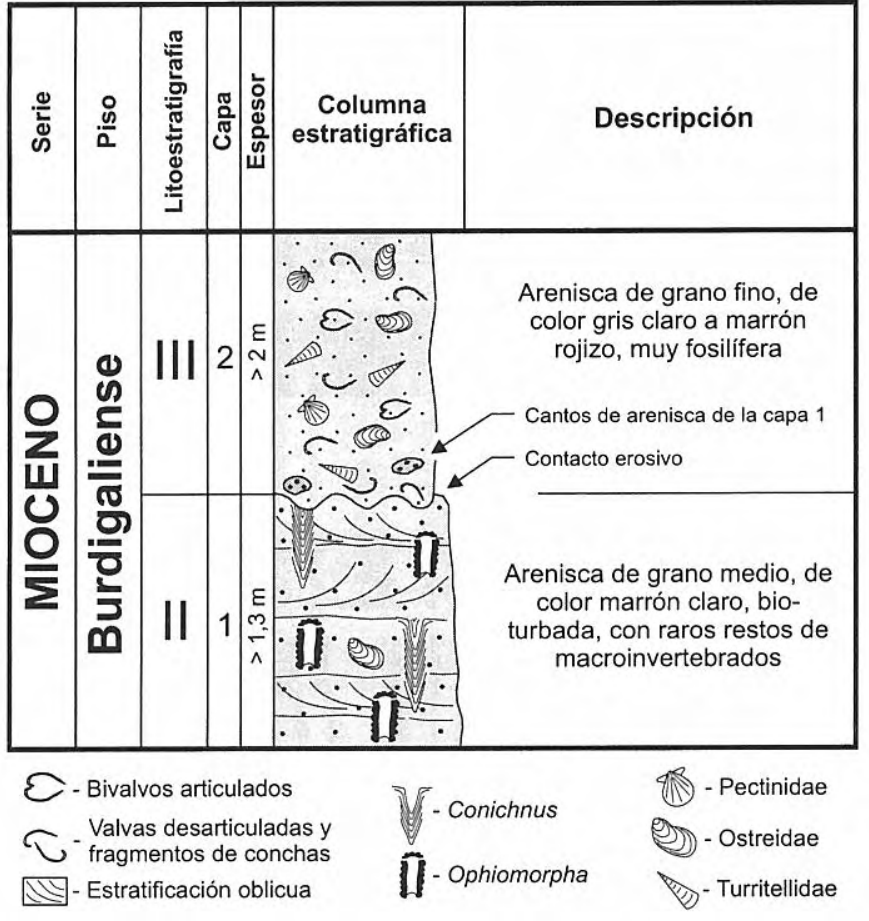

Figura 2. Perfil estratigráfico del afloramiento de CristoRei.

Calcidiscus leptoporus, Coccolithus pelagicus, Coronocyclus nitescens, Cyclicargolithus floridanus, Dictyococcites antarticus, D. productus, Discoaster deflandrei Bramlette y Riedel, Geminilithella jaffari, Helicosphaera ampliaperta, $H$. paleocarteri Theodoridis, $H$. carteri, $H$. scissura Miller, $H$. vedderi Bukry, Pontosphaera discopora Schiller, P. multipora (Kamptner) Roth, Reticulofenestra minuta Roth, $R$. gr. haqii- minutula, Sphenolithus heteromorphus, $S$. neoabies Bukry y Bramlette, Syracosphaera sp.

Aunque estratigráficamente la Unidad II de Cotter ocupa una posición subyacente a la Unidad III, en términos bioestratigráficos las asociaciones de nanofósiles calcáreos encontradas en el techo de la Unidad II y la base de la Unidad III, en los dos afloramientos muestreados, no permiten diferenciarlas y ambas quedan encuadradas en el mismo intervalo biozonal. Así, la coexistencia de Helicosphaera ampliaperta y de Sphenolitus heteromorphus permite situar todos los niveles muestreados en la biozona NN4 de Martini (1971), equivalente a la CN3 de Okada y Bukry (1980) (Fig. 3). Desde el punto de vista biocronológico, esta biozona corresponde aproximadamente al intervalo temporal comprendido entre los 18,2 y los 15,6 Ma, según la escala geocronológica de Cande y Kent (1992), lo que corresponde al Burdigaliense superior y el tránsito al Langhiense.

\section{CONCLUSIÓN BIOESTRATIGRÁFICA}

La situación bioestratigráfica presentada aquí sugiere tres cuestiones principales:

1) El intervalo temporal representado por la superficie de truncación (hiato erosivo) que separa las Unidades II y III de Cotter es inferior a la capacidad de resolución bioestratigráfica de los nanofósiles calcáreos.

2) Si la situación bioestratigráfica propuesta 

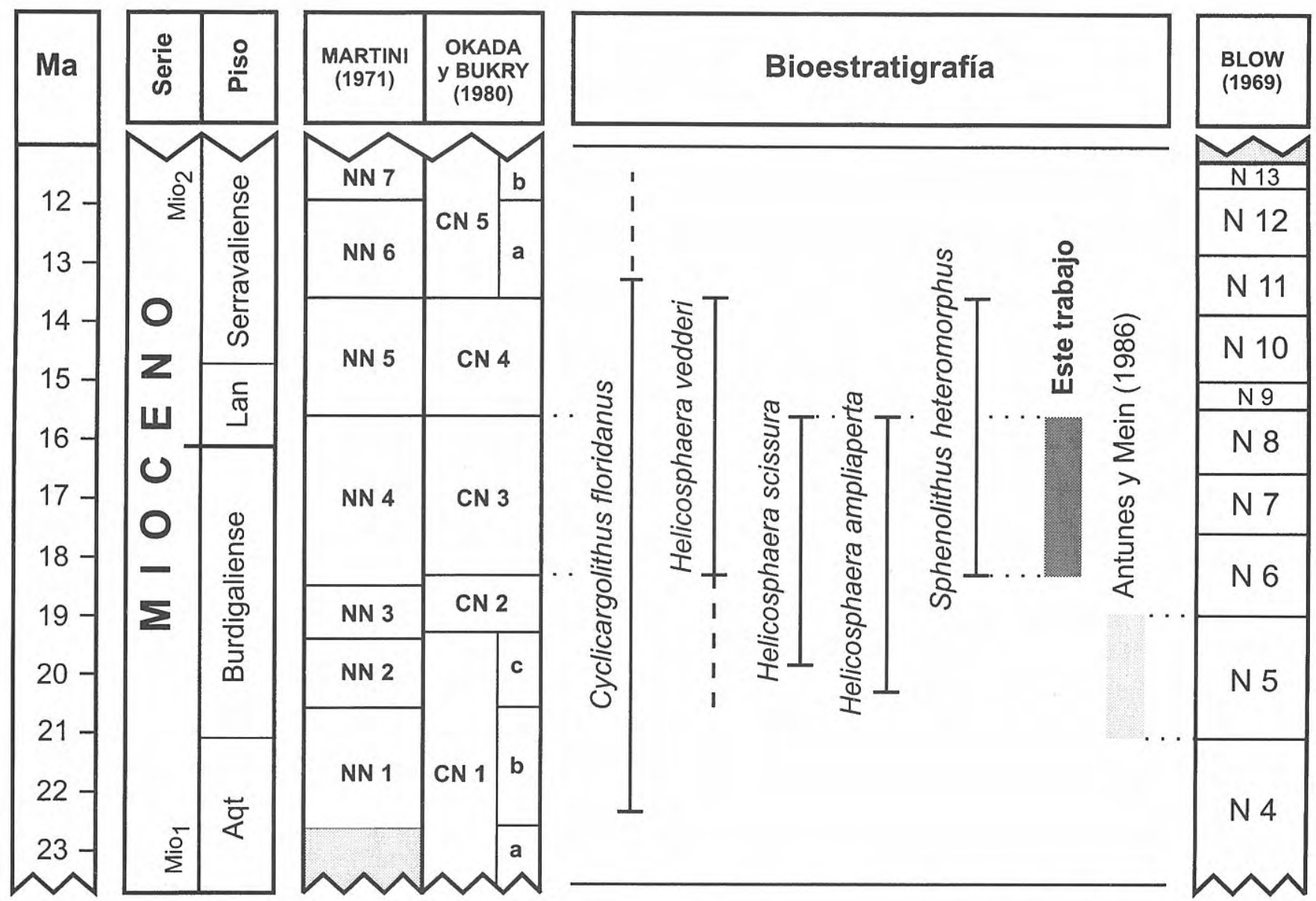

Figura 3. Distribución estratigráfica de algunas especies de nanofósiles calcáreos y situación estratigráfica del tránsito entre las Unidades II y III de Cotter en el perfil de Cristo-Rei, comparado con el dado por Antunes y Mein (1986).

anteriormente por Antunes y Mein (1986) basada en foraminíferos planctónicos (N5 de Blow, entre 21 y 19 Ma según la escala geocronológica de Cande y Kent, 1992, lo que corresponde al Burdigaliense inferior a medio) fuera representativa de las últimas fases de sedimentación de la Unidad II en la región de Lisboa, existiría un acentuado diacronismo entre los niveles superiores de esta unidad en la región de Lisboa y los que han sido atribuídos en el margen izquierdo del Tajo, en el sector de Cristo-Rei/Portinho da Costa.

3) Si la posición bioestratigráfica propuesta por Antunes y Mein (1986) fuera válida por lo menos para los niveles inferiores de la Unidad II, la extensión temporal de esta unidad debería ser mayor de la que hasta ahora ha sido admitida (ver Antunes et al., 1992), ya que sus niveles superiores (en el sector Cristo-Rei/Portinho da Costa) presentan una edad burdigaliense superior a langhiense.

\section{PALEOICNOLOGÍA SISTEMÁTICA}

Ophiomorpha Lundgren, 1891

Ophiomorpha nodosa Lundgren, 1891

Lám. I, fig. 2

El icnogénero Ophiomorpha incluye sistemas de madrigueras con frecuentes ramificaciones y caracterizadas por poseer un revestimiento constituído por elementos peletoidales arcillosos. Por su parte interior, el revestimiento es liso mientras que en el exterior presenta un aspecto tuberculado que corresponde a los elementos peletoidales. Las características de estos tubérculos permiten la diferenciación a nivel específico (Frey et al., 1978; Frey y Howard, 1985). Los ejemplares de Ophiomorpha de Cristo Rei permiten su atribución a la icnoespecie O. nodosa por la morfología redondeada de los tubérculos, que no muestran ninguna ordenación

\section{Lámina I}

1 Aspecto del afloramiento de Cristo-Rei mostrando las areniscas laminadas con Ophiomorpha nodosa y Conichnus conicus de la Unidad II de Cotter, en la parte inferior, y las areniscas muy fosilíferas de la Unidad III, en la parte superior. Escala $=10 \mathrm{~cm}$.
2 Detalle de Ophiomorpha nodosa. Escala $=5 \mathrm{~cm}$.

3 Detalle de un ejemplar de Conichnus conicus. Escala=10 $\mathrm{cm}$.

4 Detalle de un ejemplar de Conichnus conicus. Escala=10 $\mathrm{cm}$. 
Lámina I
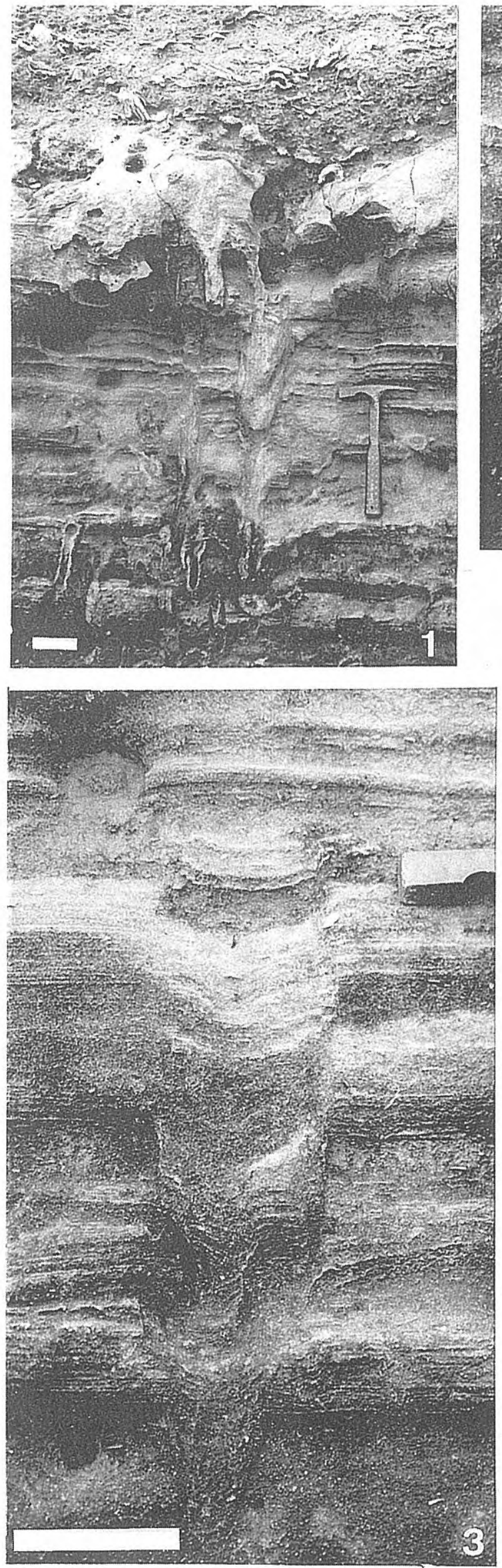
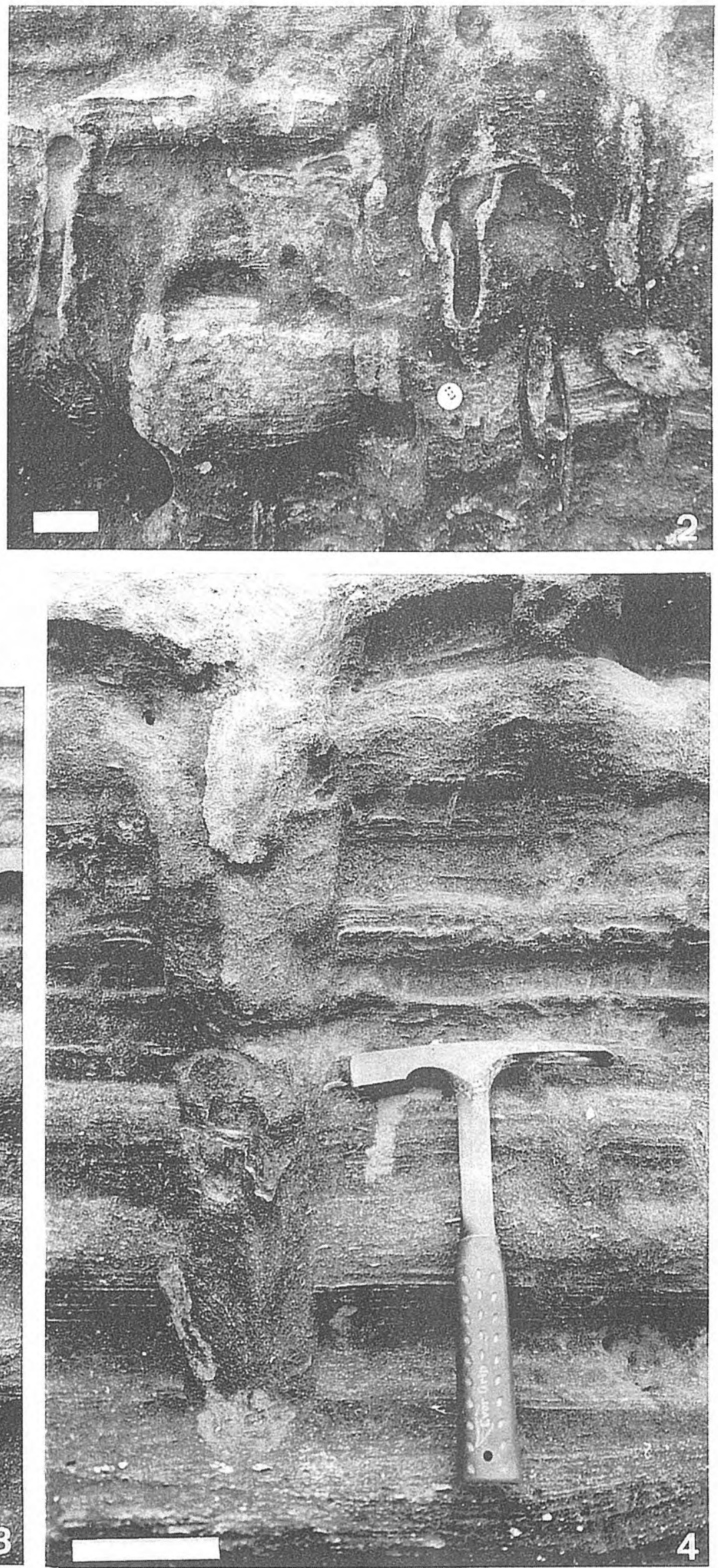
preferencial. El revestimiento es grueso (alrededor de 7 $\mathrm{mm}$ ) y muestra el aspecto externo tuberculado. A diferencia del encajante y el relleno, que son arenosos, el revestimiento está constituído por sedimento más fino de color blanco. El diámetro interno de los pozos y galerías varía entre 1,5 y $3 \mathrm{~cm}$. La configuración de los sistemas no ha podido ser observada con claridad en el campo y, aunque se observan tanto galerías horizontales como pozos verticales, ambos aparecen bastante aislados, sugiriendo que el sistema no era muy complejo ni denso.

Ophiomorpha tiene análogos directos en las madrigueras de ciertos crustáceos decápodos actuales. Es bien conocida la identidad entre Ophiomorpha y los sistemas de madrigueras de Callichirus major (Say) (anteriormente Calianassa major Say) (Weimer y Hoyt, 1964; Frey et al., 1978). Se conocen también otras especies de thalassínidos que construyen sistemas de galerías abiertos, con revestimiento peletoidal, similares a Ophiomorpha, así como especies de otros grupos de decápodos que utilizan pelets para reforzar algunas partes de sus madrigueras, generalmente chimeneas por encima de la superficie del sedimento (Frey et al., 1978; Bromley, 1990). En el registro fósil se conocen restos de thalassínidos conservados en el interior de Ophiomorpha (e.g. Beikirch y Feldman, 1980). Por todo ello, este icnogénero se atribuye a crustáceos decápodos y particularmente a los de la familia Thalassinidae.

Ophiomorpha es un icnofósil muy característico y frecuente en depósitos arenosos marinos someros, desde el Mesozoico hasta la actualidad (Frey et al., 1978; Pollard et al., 1993). Sin embargo, también se conoce en depósitos más profundos asociado a niveles episódicos arenosos (e.g. Crimes, 1977). Aunque existen algunas citas en depósitos no marinos, diversos autores han demostrado que corresponden a confusión con otros icnotaxones (Goldring y Pollard, 1995) o a colonizaciones marinas sobre materiales no marinos en situaciones transgresivas (Asgaard y Bromley, 1974).

\section{Conichnus Myannil, 1966 Conichnus conicus Myannil, 1966 Lám. I, figs. 3-4}

El icnogénero Conichnus corresponde a estructuras cónicas o troncocónicas con la base redondeada, perpendiculares a la estratificación y cuyo relleno presenta generalmente estructuras laminares ("spreiten") en forma de espiga producidas por la migración de la madriguera. Se conocen dos icnoespecies (Pemberton et al., 1988): C. conicus y C. papillatus (Myannil, 1966). La ausencia de una protuberancia basal (característica diagnóstica de C. papillatus) permite determinar los especímenes de Cristo Rei como pertenecientes a la icnoespecie C. conicus. Su diámetro es de unos $10 \mathrm{~cm}$, mientras que alcanzan longitudes de hasta $75 \mathrm{~cm}$. El relleno de estas estructuras es arenoso como el encajante y muestra láminación interna en morfologia de $\mathrm{V}$ o espiga que reproduce los cambios granulométricos y de color de la arenisca encajante. Algunos autores han puesto de manifiesto la similitud entre estructuras tipo Conichnus y estructuras de deformación física del sedimento por escape de aguas en relación al colapso de sistemas de madrigueras del tipo Ophiomorpha (Curran y Frey, 1977; Pollard et al., 1993). En el presente caso, las dimensiones grandes y la ausencia, en la mayoría de los casos, de Ophiomorpha por debajo de las estructuras, demuestra que efectivamente son estructuras de origen orgánico.

Conichnus es interpretado como una estructura de habitación de anémonas (Frey y Howard, 1981, Pemberton y Jones, 1988) por su similitud con estructuras producidas por actiniarios modernos (Shinn, 1968). Las láminas internas corresponden a "spreiten" retrusivos producidos por el organismo al desplazar su madriguera hacia arriba en respuesta a una tasa de sedimentación elevada.

Las referencias a Conichnus en la literatura son menos frecuentes que las de Ophiomorpha. Todas ellas corresponden a apariciones en depósitos arenosos sublitorales en condiciones de alta energía e importante tasa de sedimentación (Shinn, 1968; Curran y Frey, 1977; Jones y Pemberton, 1989; Pollard et al., 1993).

\section{ICNOFÁBRICA}

El análisis de icnofábricas constituye actualmente un método en auge que ofrece unos resultados más completos en el estudio de las interacciones entre el substrato y los organismos que lo habitan en medios antiguos (Goldring, 1995). El concepto de icnofábrica fue definido por Ekdale y Bromley (1983) como todos aquellos aspectos de una textura sedimentaria derivados de la bioturbación y la bioerosión a todas las escalas. La icnofábrica es un registro de las condiciones sedimentarias primarias, de la estructura de la comunidad endobentónica original y de la posterior historia tafonómica de una o más fases de actividad biogénica (Taylor y Goldring, 1993). El estudio de las icnofábricas se debe fundamentar en el análisis de varios aspectos: a) la composición icnotaxonómica de la asociación de pistas fósiles, que permitirá evaluar la icnodiversidad y el tipo de comunidad que ha dado lugar a la icnofábrica; b) las relaciones de sobreimposición entre diferentes icnotaxones ("cross-cutting relationships") y de éstos con las estructuras sedimentarias, que permitirán establecer el perfil de la comunidad endobentónica ("tier profile"), la posible ordenación en el tiempo de diferentes icnocenosis y el reconocimiento de superficies de colonización (Frey y Goldring, 1992); c) las características de conservación de las pistas fósiles -límites abruptos o irregulares, presencia de revestimiento, existencia de marcas de excavación (bioglifos), estructuras de colapso, etc.- que permitirán caracterizar la consistencia del substrato en que se produjo la icnofábrica; d) evaluación semicuantitativa del grado de bioturbación (Droser y Bottjer, 1986; Taylor y Goldring, 1993) que permitirá valorar la intensidad de la actividad biogénica en relación a la tasa de sedimentación. El análisis de todos estos aspectos 
permite alcanzar una visión integrada de los aspectos físicos y biológicos que han dado lugar a una determinada icnofábrica y consecuentemente conduce a unos resultados interpretativos de tipo paleoambiental y paleoecológico.

La icnofábrica de los depósitos arenosos del afloramiento burdigaliense de Cristo Rei incluye únicamente dos icnotaxones: Ophiomorpha nodosa y Conichnus conicus. Ni Ophiomorpha ni Conichnus parecen estar relacionados con un horizonte concreto de colonización, sino que por el contrario, su presencia en diferentes niveles sugiere una colonización continua contemporánea a la sedimentación. En el caso de Conichnus, esta contemporaneidad es indiscutible por la presencia de "spreiten" de equilibrio constituídos por sedimento que reproduce los cambios texturales de las arenas encajantes. Estos "spreiten" de equilibrio fueron producidos durante la vida de un único animal y dado que pueden alcanzar casi un metro en la vertical ponen de manifiesto la elevada tasa de sedimentación. Tanto Conichnus como Ophiomorpha aparecen bastante aislados, de manera que la icnofábrica está dominada por las estructuras sedimentarias primarias que son laminaciones horizontales y cruzadas de bajo ángulo. El grado de bioturbación del sedimento es bajo y se puede estimar como correspondiendo a un Índice de Bioturbación 2 en la escala de Taylor y Goldring (1993) o a un Índice de Icnofábrica 2-3 en la de Droser y Bottjer (1986). Estas observaciones indican que la estructuración física excedía a la actividad biogénica. Esta situación es coherente con un medio de elevada energía y elevada tasa de sedimentación, como el que corresponde a los depósitos groseros laminados que albergan la icnofábrica. La baja icnodiversidad es coherente con este tipo de ambiente sedimentario en que solamente podían prosperar organismos infaunales relativamente profundos, que podían permanecer aislados de la inestabilidad del fondo (como los decápodos productores de Ophiomorpha) u organismos semiinfáunicos con gran capacidad de reacción y reajuste ante la elevada tasa de sedimentación (como las anémonas productoras de Conichnus). Otros organismos infaunales más someros probablemente no pudieron establecerse o si lo hicieron, los productos de su actividad biogénica fueron destruídos por el efecto de la energía sobre el sedimento del fondo. Tanto Ophiomorpha como Conichnus (y sus equivalentes actuales) son estructuras biogénicas típicas de medios sublitorales someros, con substratos móviles y de elevada energía (e.g. Shinn, 1968; Jones y Pemberton, 1989; Pollard et al., 1993). La icnofábrica aquí descrita, caracterizada por su litología arenosa, el dominio de estructuras sedimentarias indicativas de elevada energía y la presencia de Conichnus con estructuras de equilibrio y Ophiomorpha, ha sido descrita también por otros autores en localidades de diferentes edades (Curran y Frey, 1977; Jones y Pemberton, 1989; Curran, 1992; Pollard et al., 1993). En todos los casos, la aparición de esta icnofábrica corresponde a situaciones de bancos arenosos ("shoals") en condiciones de energía importante, asociados a mareas o ambientes estuarinos. En el caso de Cristo Rei, la situación paleoambiental debió corresponder tambien a bancos en un medio marino sublitoral somero.

\section{CONCLUSIONES}

El estudio detallado de las características icnológicas de un depósito sedimentario puede aportar datos complementarios a los datos estrictamente sedimentológicos y paleontológicos, que completan y permiten la reconstrucción de los ambientes sedimentarios antiguos. En el afloramiento burdigaliense de Cristo Rei (Almada, Portugal) se han identificado dos icnoespecies, Ophiomorpha nodosa y Conichnus conicus, que dan lugar a una icnofábrica muy particular en la que las pistas fósiles aparecen discretas y aisladas, enmarcadas en un encajante arenoso con laminación primaria de origen sedimentario. Esta icnofábrica ha sido descrita por otros autores en depósitos de edades y localizaciones distintas, pero siempre correspondientes a unas condiciones paleoambientales muy similares. La icnofábrica de Conichnus/Ophiomorpha parece tener un importante valor paleoambiental como indicadora de bancos arenosos en medios marinos sublitorales someros de elevada energía y tasa de sedimentación.

\section{AGRADECIMIENTOS}

Este artículo supone la contribución número 12 del Grupo PALEO-Grupo de Paleontologia do Museu Nacional de História Natural da Universidade de Lisboa. Este trabajo ha sido financiado por las acciones integradas hispanoportuguesas HP95-46 y HP96-60, así como por el proyecto de investigación PB94-0946 de la DGICYT. Los autores agradecen a Marta Solsona (Universitat de Barcelona) su colaboración en el trabajo de campo, así como a los revisores, Eduardo Mayoral (Universidad de Huelva) y Jordi Martinell (Universitat de Barcelona), y a la editora de la revista María Luisa Martínez Chacón, sus comentarios sobre el manuscrito original.

\section{BIBLIOGRAFÍA}

Antunes, M.T. et Mein, P. 1986. Petits mammifères du Burdigalien inféreur (Universidade Católica, Avenida do Uruguay). Ciências da Terra (UNL), 8, 123-138.

Antunes, M.T., Pais, J. and Legoinha, P. 1992. Excursion A. Neogene deposits of Lisboa and Setubal Peninsula. In: Atlantic General Events During Neogene, 1st. Congr. RCANS, Lisbon 1992, Ciências da Terra (U.N.L.), número especial 2, 29-35.

Asgaard, U. und Bromley, R.G. 1974. Sporfossiler fra den Mellen Miocaene transgression i Søby-Fasterholt omradet. Dansk Geologisk Forening, Arsskrift for 1973, 11-19.

Beikirch, D.W. and Feldmann, R.H. 1980. Decapod crustaceans from the Pflugerville Member, Austin Formation (Late Cretaceous; Campanian) of Texas. Journal of Paleontology, 54, 309-324. 
Blow, W. 1969. Late Middle Eocene to Recent planktonic foraminiferal biostratigraphy. Proceedings First International Conference on Planktonic Microfossils, Geneva 1967, 1, 199-422.

Bromley, R.G. 1990. Trace fossils. Biology and taphonomy. Unwin Hyman Ltd., London, 280 pp.

Bromley, R.G. and Asgaard, U. 1991. Ichnofacies: a mixture of taphofacies and biofacies. Lethaia, 24, 153-164.

Cande, S. and Kent, D.V. 1992. A new geomagnetic polarity time scale for the Late Cretaceous and Cenozoic. Journal of Geophysic Research, 97, 13917-13951.

Cooper, A.H. and Romano, M. 1982. The Lower Ordovician stratigraphy of the Dornes-Figueiro dos Vinhos area, central Portugal, with descriptions of Merostomichnites ichnosp. and Rosselia socialis; two previously unrecorded trace fossils. Comunicaçoes dos Serviços Geologicos de Portugal, 68, 73-82.

Cotter, J.C.B. 1956. O Miocénico marinho de Lisboa. Comunicaçoes dos Serviços Geologicos de Portugal, 36 (suppl.), 174 pp.

Crimes, T.P. 1977. Trace fossils of an Eocene deep-sea sand fan, northern Spain. In: Trace fossils 2 (Eds. T.P. Crimes and J.C. Harper), Geological Journal Special Issue, 9, 71-90.

Curran, H.A. and Frey, R.W. 1977. Pleistocene trace fossils from North Carolina (U.S.A.) and their Holocene analogues. In: Trace fossils 2 (Eds. T.P. Crimes and J.C. Harper), Geological Journal Special Issue, 9, 139-162.

Dantas, P., Santos, V.F., Lockley, M.G. and Meyer, C.A. 1994. Footprint evidence for limping dinosaurs from the Upper Jurassic of Portugal.Gaia, 10, 43-48.

Dollfus, G.F., Cotter, J.C.B. et Gomes, J.P. 1903-1904. Mollusques tertiaires du Portugal. Planches de Céphalopodes, Gastéropodes et Pélécipodes laissées par F.A. Pereira da Costa. Acompagnés d'une explication sommaire et d'une esquisse géologique. Memórias e Comunicaçoes dos Serviços Geologicos de Portugal, 34, $6 \mathrm{pp}+46 \mathrm{pp}+65 \mathrm{pp}$.

Droser, M.L. and Bottjer D.J. 1986. A semiquantitative field classification of ichnofabric. Journal of Sedimentary Petrology, 56, 558-589.

Ekdale, A.A. 1988. Pitfalls of paleobathymetric interpretations based on trace fossil assemblages. Palaios, 3, 464-472.

Ekdale, A.A. and Bromley, R.G. 1983. Trace fossils and ichnofabrics in the Kjølby Gaard Marl, uppermost Cretaceous, Denmark. Bulletin of the Geological Society of Denmark, 31, 107-119.

Ekdale, A.A., Bromley, R.G. and Pemberton, S.G. 1984. Ichnology. The use of trace fossils in sedimentology and stratigraphy. S.E.P.M. Short Course, 15, 317 pp.

Eschwege, W.L. von 1831. Memoria geognostica ou golpe de vista do perfil das estratificações das diferentes rochas, de que he composto o terreno desde a serra de Cintra na linha de Noroeste a Sudoeste até Lisboa, atravessando o Tejo até à Serra da Arrabida, e sobre a sua idade relativa. Historia e Memorias da Academia Real das Sciencias de Lisboa, 11, 253-280.

Fonseca, B. 1977. Notes sur la geologie et la Paléontologie du Miocène de Lisbonne. XVIII - Coupe de Palença, rive gauche du Tage: stratigraphie et micropaléontologie (Coccolithophoridés). Ciências da Terra (UNL), 3, 6199.

Frey, R.W. and Goldring, R. 1992. Marine event beds and recolonization surface as revealed by trace fossil analysis. Geological Magazine, 129, 325-335.

Frey, R.W. and Howard, J.D. 1981. Conichnus and Schaubcylindrichnus: redefined trace fossils from the Upper Cretaceous of the Western Interior. Journal of Paleontology, 55, 800-804.

Frey, R.W. and Howard, J.D. 1985. Trace fossils from the Panther member, Star Point Formation (Upper Cretaceous), Coal Creek Canyon, Utah. Journal of Paleontology, 59, 370-404.

Frey, R.W., Howard, J.D. and Pryor, W.A. 1978. Ophiomorpha: its morphologic and environmental significance. Palaeogeography, Palaeoclimatology, Palaeoecology, 23, 199-229.

Fürsich, F.T. 1981. Invertebrate trace fossils from the Upper Jurassic of Portugal. Comunicaçoes dos Serviços Geologicos de Portugal, 67, 153-168.

Goldring, R. 1993. Ichnofacies and facies interpretation. Palaios, 8, 403-405.

Goldring, R. 1995. Organisms and the substrate: response and effect. In: Marine palaeoenvironmental analysis from fossils (Eds. D.W.J. Bosence and P.A. Allison). Geological Society, Special Publication, 83, 151-180.

Goldring, R. and Pollard, J.E. 1995. A re-evaluation of Ophiomorpha burrows in the Wealden Group (Lower Cretaceous) of southern England. Cretaceous Research, 16, 665-680.

Jones, B. and Pemberton, S.G. 1989. Sedimentology and ichnology of a Pleistocene unconformity-bounded, shallowing-upward carbonate sequence: the ironshore formation, Salt Creek, Grand Cayman. Palaios, 4, 343 355.

Lockley, M.G., Meyer, C.A. and Santos, V.F. 1994. Trackway evidence for a herd of juvenile sauropods from the Late Jurassic of Portugal. Gaia, 10, 27-36.

Martini, E. 1971. Standard Tertiary and Quaternary calcareous nannoplankton zonation. In: Proceedings II Planktonic Conference (Ed. A. Farinacci), Rome 1970, 739-785.

Meyer, C.A., Lockely, M.G., Robinson, J.W. and Santos, V.F. 1994. A comparison of well-preserved sauropod tracks from the Late Jurassic of Portugal and the Western United States: evidence and implications.Gaia, 10, 57 64.

Myannil, R,M. 1966. O vertikalnykh norkakh zaryvaniya v Ordovikskikh izvestiyakakh Pribaltiki. In: Organizm $i$ sreda $v$ geologicheskom proshlom, Akademiya Nauk SSSR, Paleontologichesky Institut, 200-207.

Okada, H. and Bukry, D. 1980. Supplementary modification and introduction of code numbers to the low-latitude coccolith niostratigraphic zonation (Bukry, 1973, 1979). Marine Micropaleontology, 5(3), 171-187.

Pais, J.J. 1981. Contribuição para o conhocimento da vegetação miocénica da parte occidental da Bacia do Tejo. Tesis doctoral, Universidade Nova de Lisboa, 328 pp. 
Pemberton, S.G. and Jones, B. 1988. Ichnology of the Pleistocene Ironshore Formation, Grand Cayman Island, British West Indies. Journal of Paleontology, 62, 495505.

Pemberton, S.G., Frey, R.W. and Bromley, R.G. 1988. The ichnotaxonomy of Conostichnus and other plug-shaped ichnofossils. Canadian Journal of Earth Sciences, 25, 866-892.

Pollard, J.E., Goldring, R. and Buck, S.G. 1993. Ichnofabrics containing Ophiomorpha: significance in shallow-water facies interpretation. Journal of the Geological Society, London, 150, 149-164.

Ribeiro, A., Antunes, M.T., Ferreira, M.P., Rocha, R.B., Soares, A.F., Zbyszewski, G., Almeida, F.M., Carvalho, D. et Monteiro, J.H. 1979. Introduction à la Géologie Générale du Portugal. Serviços Geologicos de Portugal, Lisboa, 114 pp.

Santos, V.F., Lockley, M.G., Meyer, C.A., Carvalho, J., Galopim de Carvalho, A.M. and Moratalla, J.J. 1994. A new sauropod tracksite from the Middle Jurassic of Portugal. Gaia, 10, 5-14.

Seilacher, A. 1964. Biogenic sedimentary structures. In: Approaches to paleoecology (Eds. J. Imbrie and N. Newell). Wiley, New York, 296-316.
Seilacher, A. 1967. Bathymetry of trace fossils. Marine Geology, 5, 413-429.

Sharpe, D. 1834. On the strata in the inmediate neighbourhood of Lisbon and Oporto. Proceedings of the Geological Society of London, 1, 394-396.

Shinn, E.A. 1968. Burrowing recent lime sediments of Florida and the Bahamas. Journal of Paleontology, 42, 879-894.

Silva, C.M. da, Cachão, M., Martinell, J. e Doménech, R. 1995. Estruturas bioerosivas como indicadores de paleolitorais rochosos. O exemplo do Miocénico da Foz da Fonte (Sesimbra, Portugal). Dados preliminares. Museu e Laboratório Mineralógico e Geológico, 4, 133137.

Smith, J. 1847. On the age of the Tertiary beds of the Tagus, with a catalogue of the fossils. Quaterly Journal of the Geological Society of London, 3, 410-422.

Taylor, A. and Goldring, R. 1993. Description and analysis of bioturbation and ichnofabric. Journal of the Geological Society of London, 150, 141-148.

Weimer, R.J. and Hoyt, J.H. 1964. Burrows of Calianassa major Say, geologic indicators of littoral and shallow neritic environments. Journal of Paleontology, 38, 761767. 